\title{
Low-density polymer thin film formation in supercritical carbon dioxide
}

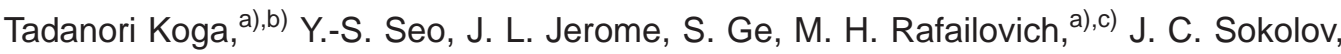 \\ and B. Chu \\ State University of New York at Stony Brook, Stony Brook, New York 11794 \\ O. H. Seeck \\ FZ Juelich GmbH, Institut für Festkoerperforschung, 52425 Juelich, Germany \\ M. Tolan \\ Universitaet Dortmund, Experimentelle Physik I, Otto-Hahn-Strasse 4, D-44221 Dortmund, Germany \\ R. Kolb \\ ExxonMobil Research and Engineering Company, Annandale, New Jersey 08801
}

(Received 24 June 2003; accepted 1 October 2003)

\begin{abstract}
We report a method for producing stable low-density polymer films by using supercritical carbon dioxide $\left(\mathrm{scCO}_{2}\right)$. Two different molecular weight polystyrene films with various thicknesses were exposed to $\mathrm{scCO}_{2}$ along the density fluctuation ridge in $P-T$ phase diagram. The swollen structures could be then frozen by flash evaporation of $\mathrm{CO}_{2}$ without forming additional voids. X-ray reflectivity data clearly showed that exposure to $\mathrm{scCO}_{2}$ could be used to produce uniform low-density films of about $2 R_{g}$ thick or less, where $R_{g}$ is radius of polymer gyration. (C) 2003 American Institute of Physics. [DOI: 10.1063/1.1629799]
\end{abstract}

Increased miniaturization in the electronics industry has produced a need for highly uniform ultrathin polymer coatings with well-controlled optical and dielectric properties. Often the thickness of these films is commensurate with a few radii of gyration $\left(R_{g}\right)$ of the polymer, and tight clearances impose very strict tolerances on surface roughness. This high degree of confinement therefore makes it difficult to satisfy the stringent uniformity requirements if we modify the electronic properties by standard methods of gas foaming or blending with other polymers or inorganic additives. Here we show an approach for producing stable, uniform, lowdensity ultrathin polymer films by using supercritical carbon dioxide $\left(\mathrm{scCO}_{2}\right)$. We demonstrate that this method provides an efficient and environmentally safe mean for producing materials with variable index of refraction and dielectric constants for electronics applications.

$\mathrm{ScCO}_{2}$ has long been known to be a "green" medium for polymer chemistry or material science. ${ }^{1}$ Unfortunately, its potential benefits have not been fully exploited since only a limited class of polymers can be dissolved in $\mathrm{scCO}_{2}$. Recently, using in situ neutron reflectivity, we have shown that a wide variety of polymer thin films can swell as much as $30 \%-60 \%$ when exposed to $\mathrm{scCO}_{2}$ within a narrow temperature and pressure regime, known as the "density fluctuation ridge" ${ }^{2-4}$ that defines the maximum density fluctuation amplitude in $\mathrm{CO}_{2}$. Furthermore, we found that the in situ film quality, i.e., density, roughness, and film thickness, could be frozen by flash evaporation of $\mathrm{CO}_{2}$. This process vitrifies the polymer without formation of micron-sized voids which are known to form in the bulk when $\mathrm{scCO}_{2}$ is forced under high pressures into polymers with which it is immiscible.,

In this letter, we report $\mathrm{x}$-ray reflectivity (XR) measure-

\footnotetext{
${ }^{a}$ Author to whom correspondence should be addressed

${ }^{b)}$ Electronic mail: tkoga@ notes.cc.sunysb.edu

${ }^{c}$ Electronic mail: mrafailovich@notes.cc.sunysb.edu
}

ments where the density profile of the vitrified films is measured directly and correlated with other properties such as the dielectric constant, index of refraction, and surface glass transition.

Samples consisted of two kinds of hydrogenated polystyrene (PS) $\left(\mathrm{PS} 1: M_{w}=2.0 \times 10^{5}, \quad \mathrm{PS} 2: M_{w}=6.5 \times 10^{5}\right)$ with glass transition temperature, $T_{g}=100^{\circ} \mathrm{C}$. Both polymers were obtained from Polymer Laboratories and their polydispersity indices were 1.05 . Thin films were spun cast on $\mathrm{HF}$ etched $\mathrm{Si}$ substrates and annealed for $5 \mathrm{~h}$ in vacuum of $10^{-6}$ Torr at $T=150^{\circ} \mathrm{C}>T_{g}$, in order to remove residual solvent and spin induced stress. The films were then placed in a high pressure chamber containing $\mathrm{scCO}_{2}$ at $T=36^{\circ} \mathrm{C}$ and $P=8.2 \mathrm{MPa}$ for $2 \mathrm{~h}$, and then quickly depressurized to atmospheric pressure within $10 \mathrm{~s}$. This condition was chosen since they correspond to the density fluctuation ridge in the vicinity of the critical point and can be still tuned within the experimental resolution. The XR measurements have been carried out at the X10B beamline of the National Synchrotron Light Source, Brookhaven National Laboratory using photon energy of $11 \mathrm{keV}$, i.e., x-ray wavelength $(\lambda)$ of $1.13 \AA$ A.

Figure 1 shows the representative XR profile for a PS1 thin film before and after exposure to $\mathrm{scCO}_{2}$. In the figure we plot the scattering intensity, $I\left(q_{z}\right)$, as a function of the momentum transfer normal to the surface, $q_{z}=4 \pi \sin \theta / \lambda$ where $\theta$ is the glancing angle of incidence. The dashed line in the figure illustrates the shift of the Kiessig fringes to a higher frequency after exposure, which indicates that the layer has thickened. A three layer model used to obtain the solid line fits to the data is shown in the inset (a), where we plot the dispersion $(\delta)$ value in the $\mathrm{x}$-ray refractive index corresponding to the silicon substrate and the native oxide and the PS layers. The dashed and solid lines correspond to the profiles before and after exposure, where we see that the 


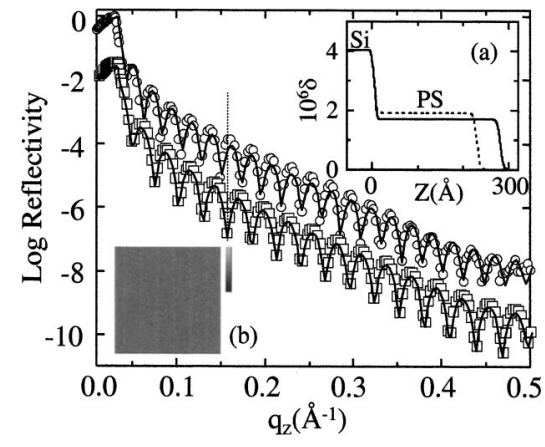

FIG. 1. Measured XR before $(\square)$ and after $(\bigcirc)$ exposure. The solid lines correspond to the best-fit to the data using the dispersion profiles shown in the inset (a). The inset (b) shows AFM data (image size of $10 \mu \mathrm{m} \times 10 \mu \mathrm{m}$, height scale of $0-5 \mathrm{~nm}$ ) for the exposed film.

initial thickness, $L_{0}=223 \AA$ has increased to $L=275 \AA$. This corresponds to a linear dilation, $S_{f}=\left(L-L_{0}\right) / L_{0}$ $=0.23$ which is much larger than the bulk swelling of $0.07 .{ }^{4}$ Examination of the data also shows that the dilation occurs without a significant damping of the Kiessig fringes. This is consistent with the fitting model where we found that the rms roughness $(\sigma)$ between the polymer and air layers remain unchanged $(\sigma=4.6 \pm 0.4 \AA)$ before and after exposure. This was further confirmed by measuring the x-ray diffuse scattering of the films where no change in intensity or deviation from the usual capillary power law decay ${ }^{7}$ was observed before and after exposure. These results show that no lateral density inhomogeneity larger than the x-ray wavelength and less than the coherence length of the x-ray beam $(1 \mu \mathrm{m})$ occurs due to exposure in $\mathrm{scCO}_{2}$. In order to further check that large voids $(>1 \mu \mathrm{m})$ were not present, the surface of the film was scanned with atomic force microscopy (AFM) in series of $100 \times 100 \mu \mathrm{m}$ scans across the entire sample surface. A typical scan is shown in the inset (b) where the surface appears flat and featureless with $\sigma=5 \AA$. Consequently, it is reasonable to conclude that the films exposed to $\mathrm{CO}_{2}$ in the density fluctuating regime have an excessive amount of the free volume and can be used to produce uniform, lowdensity polymer films. In order to determine whether the decrease in density is a function of $L_{0}$ or molecular weight $\left(M_{w}\right)$, we performed similar measurements on films of different thicknesses for PS1 and PS2. The results are plotted in Fig. 2(a) where we see that the density of PS films can be decreased from approximately $23 \%$ to $13 \%$ by varying the thickness of the film. It is interesting to note that the data can be collapsed on a universal curve when $L_{0}$ is scaled by $R_{g}$, expressed in terms of the polymerization index, $N$, as $R_{g}$ $=6.7 \AA \times(N / 6)^{1 / 2}$, as shown in the inset of Fig. 2(a). Hence, when confined as a thin film on a surface, thickness as well as molecular structure influence the density. The density obtained from the in situ $S_{f}$ values ${ }^{2,4}$ is plotted as a dashed line in the inset of Fig. 2(a), assumed that the polymer chains stretch only in the direction normal to the surfaceand the total mass is conserved. ${ }^{8}$ The fact that the results from the differing experiments are in good agreement indicates that in the case of PS, rapid sublimation of the gas preserves the in situ dilation of the films.

We also exposed films that were much thicker than size of polymer chain $\left(2 R_{g}\right)$ in order to see whether dilation occurred in the absence of direct contacts with the surface. The Downloaded 21 Dec 2006 to 134.94.122.39. Redistribution subject
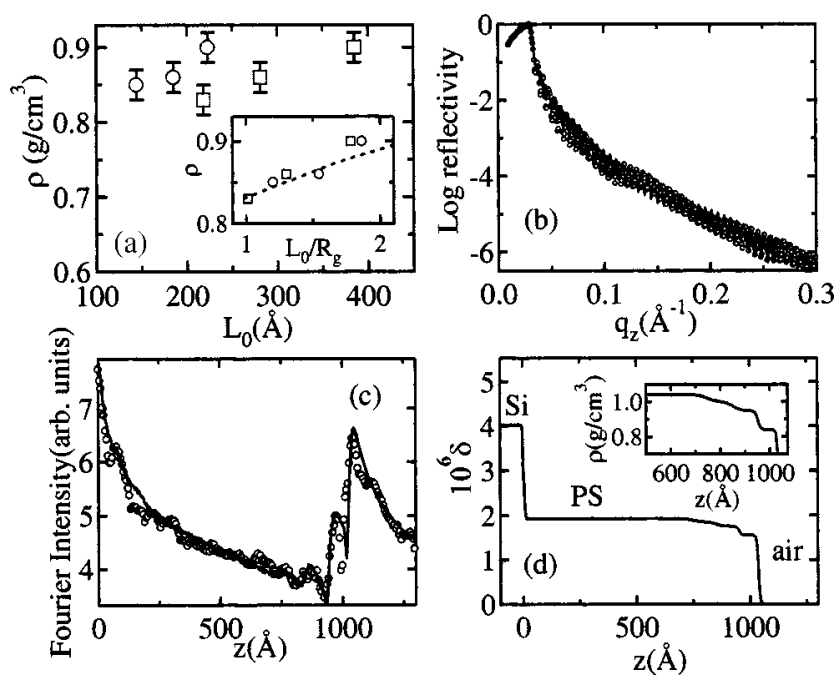

FIG. 2. (a) $\rho$ vs $L_{0}$ for the exposed films: PS1 (○), PS2 ( $\square$ ). In the inset, $L_{0} / R_{g}$ dependence of $\rho$ is shown. (b) Measured XR $(\bigcirc)$ and refinement (solid line) of the thick frozen PS1 film. (c) FT of measured (O) and calculated (solid line) XRs. (d) $\delta$ profile used for the refinement. In the inset, $\rho$ expanded near the surface area is shown.

XR spectra for the PS1 film $\left(L_{0}=930 \AA, S_{f}=0.10\right)$ are shown in Fig. 2(b) where we can see that the data shows a pronounced beating pattern. Since we can observe many Kiessig fringes, a Fourier transform (FT) analysis method ${ }^{9}$ was used to analyze the profile. The FT profile of the spectra corresponding to the data in Fig. 2(b) is shown in Fig. 2(c). ${ }^{10}$ In this technique the XR and FT data are fitted simultaneously using a dispersion model. The best fits [solid lines in Figs. 2(b) and 2(c)] were obtained with the model shown in Fig. 2(d) which consisted of six layers, silicon substrate, native oxide, and four different PS layers, based upon the number of the peaks in the FT profile. From the inset of Fig. 2(d) we can clearly see that a layer about $100 \AA$ thick is formed at the polymer/air surface with a reduced density of $\rho$ $=0.83 \mathrm{~g} / \mathrm{cm}^{3}$ and a relatively sharp interface of about $10 \AA$ with the layers beneath. The subsequent layers also have reduced density, but these layers are more diffuse and decay over a region of approximately $200 \AA$ towards a uniform layer with the bulk density. This behavior, which was independent of $M_{W}$, was observed in all films larger than $4-5 R_{g}$, and for thickness of at least $200 \mathrm{~nm}$, which are at the limit of our XR detection method. Hence, surface modification through exposure to $\mathrm{scCO}_{2}$ appears to be a universal phenomenon for all the polymer films regardless of thickness. This effect had not been previously reported since it occurs on a nanometer scale and is difficult to detect when only bulk properties are measured.

The reduction in density has several consequences regarding the material properties of the films: (i) A decrease in the density of a film can be correlated to a decrease in its optical index of refraction $\left(n_{f}\right)$ and dielectric constant. We therefore measured the corresponding changes in the electronic properties of the exposed films by using spectroscopic ellipsometry. ${ }^{11}$ Figure 3 shows the refractive indices of the PS2 layers for the unexposed $\left(L_{0}=2 R_{g}\right)$ and two exposed films with $L_{0}=1.3 R_{g}\left(S_{f}=0.21\right)$ and $L_{0}=2 R_{g}\left(S_{f}=0.16\right)$. From the figure we can clearly see that the $n_{f}$ values for the exposed films decreased with increasing the $S_{f}$ values over to AIP license or copyright, see http://apl.aip.org/apl/copyright.jsp 


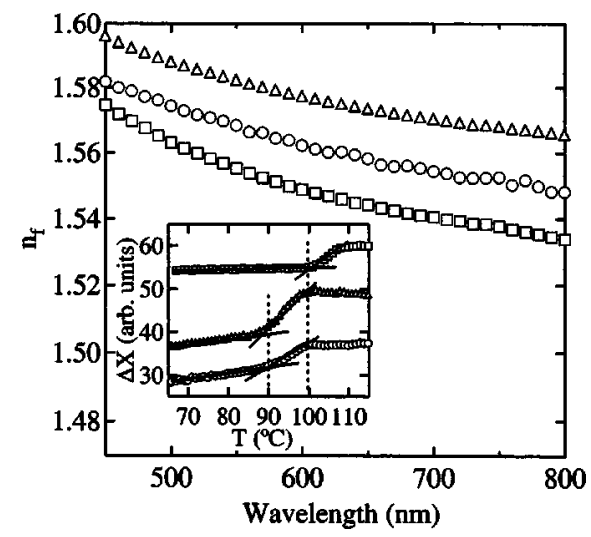

FIG. 3. Index of refraction for PS2: before $(\Delta)$ and after $\left[L_{0}=2 R_{g}(\bigcirc)\right.$ and $\left.L_{0}=1.3 R_{g}(\square)\right]$. In the inset, $\Delta X$ vs $T$ curves for PS2: exposed films $L_{0}$ $=1 R_{g}(\bigcirc)$ and $L_{0}=6.4 R_{g}(\Delta)$, and unexposed film ( $\left.\square\right)$.

the wide wavelength range of $450-800 \mathrm{~nm}$. Since dielectric constant of materials can be derived from a square of refractive index, we can therefore vary the dielectric constant for PS film from $2 \%$ to $4 \%$ in the same $L_{0}, R_{g}$ parameter space.

(ii) A change in density is also associated with a change in free volume of the polymer. It is well known from the Flory-Fox theory ${ }^{12}$ that a change in free volume will result in decreased $T_{g}$. In order to explore this hypothesis, $T_{g}$ measurements were made using the shear modulation force microscopy (SMFM) technique using a Digital Instruments Dimension 3000 which was previously shown to be a simple and robust method for measuring the surface $T_{g}$ with an accuracy of $2{ }^{\circ} \mathrm{C} .{ }^{13}$ In the inset of Fig. 3 we plot the modulation amplitude, $\Delta X$, vs $T$ for an unexposed PS2 film and two PS2 films exposed to $\mathrm{scCO}_{2}$ one, $L_{0}=220 \AA$ or $1 R_{g}$ thick, and another $1400 \AA$ or $6.4 R_{g}$ thick. From the figure we can see that the discontinuity in the plot, which corresponds to $T_{g}$, occurs at $T=100{ }^{\circ} \mathrm{C}$ or the bulk $T_{g}$ for the unexposed film, as expected. The $T_{g}$ value for the exposed film $1 R_{g}$ thick $\left(S_{f}=0.23\right)$, decreases by $10{ }^{\circ} \mathrm{C}$ compared to that of the unexposed PS film. Similarly, for the film $6.4 R_{g}$ thick shown by squares, we also find a $T_{g}$ value of $90^{\circ} \mathrm{C}$. This result is consistent with the x-ray data which show a region of effective dilation, $S_{f} \sim 0.2$ near the surface, since SMFM is a surface sensitive technique it cannot detect the difference in the concentration profiles of the thick and thin films below the surface.
In conclusion we have found that exposure to $\mathrm{scCO}_{2}$ in the $P-T$ range corresponding to a density fluctuation regime, can be used to produce uniform low-density films of approximately $2 R_{g}$ or less. For films $L_{0} \gg 2 R_{g}$, a region of low-density, about $3 R_{g}$ thickness, is produced at the surface that then decays into the bulk. In this study we used PS, where the miscibility of $\mathrm{scCO}_{2}$ is low and the dilation is enhanced only by a factor of 0.2 at the ridge. It is expected that much higher dilations can be achieved in polymers such as Teflon and the acrylates. ${ }^{4}$ This technique can therefore be exploited to produce high quality films of low dielectric constants, variable index of refraction, and enhanced adhesion at lower temperatures to other surfaces.

Support of this work by the NSF-MRSEC is gratefully acknowledged.

${ }^{1}$ M. A. McHugh and V. Krukonis, Supercritical Fluids Extraction Principles and Practice (Woburn, MA, 1994).

${ }^{2}$ T. Koga, Y.-S. Seo, Y. Zhang, K. Shin, K. Kusano, K. Nishikawa, M. H. Rafailovich, J. C. Sokolov, B. Chu, D. Peiffer, R. Occhiogrosso, and S. K. Satija, Phys. Rev. Lett. 89, 125506 (2002).

${ }^{3}$ T. Koga, Y.-S. Seo, X. Hu, K. Shin, Y. Zhang, M. H. Rafailovich, J. C. Sokolov, B. Chu, and S. K. Satija, Europhys. Lett. 60, 559 (2002).

${ }^{4}$ T. Koga, Y.-S. Seo, K. Shin, Y. Zhang, M. H. Rafailovich, J. C. Sokolov, B. Chu, D. Peiffer, and S. K. Satija, Macromolecules 36, 5236 (2003).

${ }^{5}$ K. A. Arora, A. J. Lesser, and T. J. McCarthy, Macromolecules 31, 4614 (1998).

${ }^{6}$ C. M. Stafford, T. P. Russell, and T. J. McCarthy, Macromolecules 32, $7610(1999)$

${ }^{7}$ J. Wang, M. Tolan, O. H. Seeck, S. K. Sinha, O. Bahr, M. H. Rafailovich, and J. Sokolov, Phys. Rev. Lett. 83, 564 (1999).

${ }^{8}$ As we already reported [T. Koga, Y.-S. Seo, X. Hu, K. Shin, Y. Zhang, M. H. Rafailovich, J. C. Sokolov, B. Chu, and S. K. Satija, Europhys. Lett. 60, 559 (2003)] a roughly $20 \AA$ decrease cannot be avoided in the quick evaporation process. We therefore estimated the "apparent" dilation for the films considering this change to calculate the density.

${ }^{9}$ O. H. Seeck, I. D. Kaendler, M. Tolan, K. Shin, M. H. Rafailovich, J. C. Sokolov, and R. Kolb, Appl. Phys. Lett. 75, 2713 (2000).

${ }^{10} \mathrm{FT}$ profile is related to the corresponding one-dimensional Patterson function, i.e.:

$F\left(d, q_{1}\right)=\left|\int_{q_{1}}^{q_{2}} q_{z}^{4} I\left(q_{z}\right) \exp \left(i q_{z} d\right) d q_{z}\right|^{2}$, where $q_{1}$ is the lower $q$ limit set as $q_{1}=0.052 \AA^{-1}, q_{2}$ is the higher $q$ limit set as $q_{2}=1.0 \AA^{-1}$ and $d$ is the film thickness, respectively.

${ }^{11}$ A H-VASE spectroscopic ellipsometry (J. A. Woollam Co., Inc.) was used. In order to fit the data, the same three-layer model was used with the literature $n_{f}$ values for native oxide and Si substrate and the film thicknesses determined by XR.

${ }^{12}$ T. G. Fox and P. J. Flory, J. Appl. Phys. 21, 581 (1950).

${ }^{13}$ S. Ge, Y. Pu, W. Zhang, M. H. Rafailovich, J. C. Sokolov, C. Buenviaje, R. Buckmaster, and R. M. Overney, Phys. Rev. Lett. 85, 2340 (2002). 